DAMTP-95-72

hep-th/9601016

\title{
Abelian duality in WZW models
}

\author{
Matthias R. Gaberdiel \\ Department of Applied Mathematics and Theoretical Physics \\ University of Cambridge, Silver Street \\ Cambridge, CB3 9EW, U. K.
}

December 1995

\begin{abstract}
We analyse abelian $T$-duality for WZW models of simply-connected groups. We demonstrate that the dual theory is a certain orbifold of the original theory, and check that it is conformally invariant. We determine the spectrum of the dual theory, and show that it agrees with the spectrum of the original theory.
\end{abstract}

\footnotetext{
${ }^{1}$ e-mail: M.R.Gaberdiel@damtp.cam.ac.uk
} 


\section{Introduction}

Recently there has been a renewed interest in duality transformations in field theory and string theory [19, 21, 18]. These transformations relate the strong coupling region of one theory to the weak coupling regime of another theory, the dual theory. The former is typically not accessible by perturbation theory, whereas the latter might well be. Duality therefore seems to provide a very promising tool for the non-perturbative analysis of field theories and string theories.

The archetypal prototype of this transformation in string theory, is what is now usually referred to as $T$-duality. This is the transformation which exchanges the winding and momentum modes of a free boson whose target space is a circle. The transformation also maps the radius $R$ of the circle to its inverse, and thus relates phenomena at large and small scales.

In this paper we want to study a slightly more complicated variation of this transformation in detail, a chiral abelian duality transformation of a WZW model. In this context the rôle of the target space circle is played by the Cartan torus of a simple group. This torus is embedded in the group in a rather non-trivial fashion, and, as a consequence, the analysis of the duality transformation is much more involved. On the other hand, these models are still sufficiently simple to allow a detailed analysis. In particular, both the original and the dual theory possess infinite dimensional symmetry algebras, and the theories are therefore essentially determined by the representation theory of these algebras. For example, it is rather easy to see directly that the dual theory is conformally invariant, and the calculation of the spectrum is a fairly tractable algebraic problem.

In the archetypal situation, it was clear how to define the duality transformation from the structure of the spectrum of the theory. In the situation at hand, this is not the case, and we therefore follow the procedure of Buscher [5, 17] to derive an action for the dual theory from the classical action. We then quantise this action, following Witten [20]. It turns out that the dual theory has two commuting infinite dimensional symmetry algebras, a (right-moving) untwisted Kac-Moody algebra, and a (left-moving) twisted Kac-Moody algebra, whose (inner) twists we determine. As the twist is inner, the twisted algebra is isomorphic to the untwisted algebra.

The classical action has typically more than one quantisation [11], and we want to analyse whether there is one such quantisation for which the spectrum of the dual theory reproduces the original spectrum. This is a rather non-trivial problem, as the spectrum of a twisted algebra typically differs from the spectrum of the corresponding untwisted algebra, even if they are related by inner twists and thus isomorphic as algebras. In the case at hand, however, due to the rather special form of the twists, such a quantisation may be found. The resulting dual theory (which is then equivalent to the original theory) can be naturally interpreted as an orbifold of the original theory.

It should be mentioned that these models have already been considered in [15, 1] (for a review see also [4]), where, however, the structure of the dual theory was not analysed in detail. Furthermore, in [15] the global structure of the (target space) group was not properly taken into account, and as a consequence, the duality transformation was misidentified with a Weyl transformation (see also [3]). 
The paper is organised as follows. In section 2, we derive the action for the dual theory, paying particular attention to the global properties of the target space group. We then quantise this action in section 3, and determine the twist of the left-moving KM algebra. In section 4, we construct the quantisation for which the spectrum is preserved, and section 5 contains some concluding remarks.

\section{The duality transformation}

Let us consider the WZW action [20, 8, 6]

$$
S_{0}[g]=-\frac{k}{4 \pi} \int_{\mathcal{M}} d z d \bar{z} \operatorname{Tr}\left(g^{-1} \partial g g^{-1} \bar{\partial} g\right)+\frac{k}{12 \pi} \int_{\mathcal{B}} d^{3} y \varepsilon^{i j k} \operatorname{Tr}\left(g^{-1} \partial_{i} g g^{-1} \partial_{j} g g^{-1} \partial_{k} g\right),
$$

where $g: \mathcal{M} \rightarrow G, \mathcal{M}$ is a closed two-dimensional manifold, and $\mathcal{B}$ is a three-dimensional manifold with boundary $\mathcal{M}$. The trace is the Killing form on the algebra $\mathfrak{g}$ of the Lie group $G$, normalised so that the long roots have length square equal to 2 . We shall only consider the case where $G$ is simply connected; then the quantisation condition on $k$ is that it is an integer. We want to parametrise $g_{0}$ as

$$
g_{0}=e^{i \theta^{\alpha} e_{\alpha}} g,
$$

where the sum extends from $\alpha=1$ to $n$, and the $e_{\alpha}$ are a basis for a ( $n$-dimensional) subalgebra of a Cartan subalgebra. Here $n \leq r$, the rank of the Lie group $G$, the $\theta^{\alpha}$ are allowed to vary independently, and $g$ lies in some coset of $G$. We could also consider the case, where the Cartan torus is multiplied from the right, and this would lead to a completely analogous discussion.

The manifold $\mathcal{M}$ need not be simply-connected and can in fact be of any genus. Here we shall mainly consider the case where $\mathcal{M}$ is a torus, as this is sufficient for the determination of the spectrum. Typically, the fields $\theta^{\alpha}$ have then non-trivial winding, and we denote by $\Delta^{c} \theta^{\alpha}$ the monodromy of $\theta^{\alpha}$ along the cycle $c$, where $c=a$ or $c=b$, the two non-trivial cycles of the torus. The consistency condition is then

$$
\frac{1}{2 \pi} \sum_{\alpha=1}^{n} \Delta^{c} \theta^{\alpha} e_{\alpha} \in R^{*},
$$

where $R^{*}$ is the coroot lattice of the Lie algebra. (If $G$ were not simply-connected, the lefthand side of (2.3) would only have to be in the coweight lattice.) As the $\theta^{\alpha}$ are independent variables, this should imply that for each $\theta^{\alpha}$ there exists a minimal monodromy $\Delta^{m} \theta^{\alpha}$ such that

$$
\frac{1}{2 \pi} \Delta^{m} \theta^{\alpha} e_{\alpha} \in R^{*}
$$

is a generating element of the coroot lattice (i.e. no shorter multiple of it is in the coroot lattice), and all other monodromies are integer multiples of the minimal one. To simplify notation, we shall thus assume that the $e_{\alpha}$ are roots of the Lie algebra $\mathfrak{g}$. Then (2.4) is equivalent to

$$
\Delta^{m} \theta^{\alpha}=2 \pi \frac{2}{\operatorname{Tr}\left(e_{\alpha} e_{\alpha}\right)}
$$


We want to use the Polyakov-Wiegmann property (PW) [16]

$$
S_{0}\left[g_{1} g_{2}\right]=S_{0}\left[g_{1}\right]+S_{0}\left[g_{2}\right]-\frac{k}{2 \pi} \int_{\mathcal{M}} d z d \bar{z} \operatorname{Tr}\left(g_{1}^{-1} \bar{\partial} g_{1} \partial g_{2} g_{2}^{-1}\right)
$$

to rewrite the action as

$$
S_{0}\left[g_{0}\right]=S_{0}[g]+\frac{k}{4 \pi} \int_{\mathcal{M}} d z d \bar{z} d_{\alpha \beta} \partial \theta^{\alpha} \bar{\partial} \theta^{\beta}-\frac{k}{4 \pi} \int_{\mathcal{M}} d z d \bar{z} J_{\alpha} \bar{\partial} \theta^{\alpha}
$$

where

$$
d_{\alpha \beta}=\operatorname{Tr}\left(e_{\alpha} e_{\beta}\right), \quad \text { and } \quad J_{\alpha}=2 i \operatorname{Tr}\left(\partial g g^{-1} e_{\alpha}\right) .
$$

It should be noted that this simple form of the (PW) property only holds if $\mathcal{M}$ is simplyconnectede; in general it has to be modified by the inclusion of a topological term involving $g_{1}$ and $g_{2}$ [8, Appendix 2, eq. (10)]. For example, we can calculate the action for $g_{1} g_{2}$, where $g_{j}=\exp \left(i \theta^{j} e_{j}\right)$ is in the Cartan torus, in two different ways, directly from the action, and using the $(\mathrm{PW})$ formula

$$
S_{0}^{(P W)}\left[g_{1} g_{2}\right]-S_{0}\left[g_{1} g_{2}\right]=\frac{k}{4 \pi} \int_{\mathcal{M}} d z d \bar{z} \operatorname{Tr}\left(e_{1} e_{2}\right)\left(\bar{\partial} \theta^{1} \partial \theta^{2}-\bar{\partial} \theta^{2} \partial \theta^{1}\right) .
$$

The difference is a topological term which vanishes for simply-connected world-sheets. If the world-sheet has the topology of a torus, however, we can integrate by parts to obtain

$$
S_{0}^{(P W)}\left[g_{1} g_{2}\right]-S_{0}\left[g_{1} g_{2}\right]=\frac{\operatorname{Tr}\left(e_{1} e_{2}\right) k}{4 \pi}\left(\Delta^{a} \theta^{1} \Delta^{b} \theta^{2}-\Delta^{b} \theta^{1} \Delta^{a} \theta^{2}\right)
$$

(2.4) now implies that this is in $\pi k \mathbb{Z}$, as the Killing form of two elements in the coroot lattice is integral, but it is not always in $2 \pi \mathbb{Z}$. On the other hand, it should be clear that a topological term involving $e^{i \theta^{\alpha} e_{\alpha}}$ and $g$ will not affect the following argument. (Such a term will only restrict the allowed monodromies for $g-c f$. the discussion after (2.21).)

Originally, the duality transformation was formulated in terms of gauging the action and fixing the gauge by introducing Lagrange multipliers. Upon integrating out the Lagrange multipliers, the gauge fields are restricted to be flat, and we can choose the monodromies of the Lagrange multipliers, so that the monodromies of the gauge fields lie in a lattice. For compact gauge groups we can thus guarantee that the gauge fields are trivial, and the original action can be recovered. On the other hand, integrating out the gauge fields leads to a new action in which the Lagrange multipliers take the rôle of the fields whose symmetry was gauged [5, 1, 17].

It was realised recently [3] that the duality transformation can also (formally) be obtained as a certain canonical transformation. In this approach, it is not necessary to gauge the action, but the transformation with respect to $\theta^{1}$, say, is simply the transformation

$$
\int D \theta^{1} \exp \left(\frac{\lambda k i}{4 \pi} \int_{\mathcal{M}} d z d \bar{z}\left(\bar{\partial} \theta^{1} \partial \psi^{1}-\partial \theta^{1} \bar{\partial} \psi^{1}\right)\right) \exp \left(i S_{0}\left[g_{0}\right]\right)
$$

\footnotetext{
${ }^{2}$ The remark in [1, p. 81] is not quite correct.
} 
where $\lambda$ is an arbitrary constant which parametrises the freedom to rescale $\psi^{1}$. To calculate this transformation, we first rewrite the action as

$$
\begin{gathered}
S_{0}\left[g_{0}\right]=S_{0}[g]+\frac{k}{4 \pi} \int_{\mathcal{M}} d z d \bar{z}\left[d_{11} \partial \theta^{1} \bar{\partial} \theta^{1}-J_{1} \bar{\partial} \theta^{1}+\sum_{\alpha \geq 2}\left(d_{1 \alpha} \partial \theta^{1} \bar{\partial} \theta^{\alpha}+d_{\alpha 1} \bar{\partial} \theta^{1} \partial \theta^{\alpha}\right)\right] \\
+\frac{k}{4 \pi} \int_{\mathcal{M}} d z d \bar{z}\left[\sum_{\alpha, \beta \geq 2} d_{\alpha \beta} \partial \theta^{\alpha} \bar{\partial} \theta^{\beta}-\sum_{\alpha \geq 2} J_{\alpha} \bar{\partial} \theta^{\alpha}\right] .
\end{gathered}
$$

We then add the generating functional for the canonical transformation and complete the square to obtain

$$
\begin{aligned}
S_{0}\left[g_{0}\right]+ & \frac{\lambda k}{4 \pi} \int_{\mathcal{M}} d z d \bar{z}\left(\bar{\partial} \theta^{1} \partial \psi^{1}-\partial \theta^{1} \bar{\partial} \psi^{1}\right) \\
= & S_{0}[g]+\frac{k}{4 \pi} \int_{\mathcal{M}} d z d \bar{z} d_{11}\left(\partial \theta^{1}+\frac{d_{\alpha 1}}{d_{11}} \partial \theta^{\alpha}-\frac{J_{1}}{d_{11}}+\frac{\lambda}{d_{11}} \partial \psi^{1}\right)\left(\bar{\partial} \theta^{1}+\frac{d_{1 \alpha}}{d_{11}} \bar{\partial} \theta^{\alpha}-\frac{\lambda}{d_{11}} \bar{\partial} \psi^{1}\right) \\
& +\frac{k}{4 \pi} \int_{\mathcal{M}} d z d \bar{z}\left[\frac{\lambda^{2}}{d_{11}} \partial \psi^{1} \bar{\partial} \psi^{1}-\frac{\lambda}{d_{11}} J_{1} \bar{\partial} \psi^{1}\right] \\
& +\frac{k}{4 \pi} \int_{\mathcal{M}} d z d \bar{z}\left[\frac{\lambda d_{\alpha 1}}{d_{11}} \partial \theta^{\alpha} \bar{\partial} \psi^{1}-\frac{\lambda d_{1 \alpha}}{d_{11}} \partial \psi^{1} \bar{\partial} \theta^{\alpha}\right] \\
& +\frac{k}{4 \pi} \int_{\mathcal{M}} d z d \bar{z}\left[\left(d_{\alpha \beta}-\frac{d_{\alpha 1} d_{1 \beta}}{d_{11}}\right) \partial \theta^{\alpha} \bar{\partial} \theta^{\beta}-\left(J_{\alpha}-\frac{d_{1 \alpha}}{d_{11}} J_{1}\right) \bar{\partial} \theta^{\alpha}\right]
\end{aligned}
$$

where a summation over $\alpha$ and $\beta$ from 2 to $n$ is implicit. We shift the variable of integration $\theta^{1}$, without incurring a Jacobian factor, to obtain a purely quadratic term in $\theta^{1}$. Upon integration this gives only a constant, and the dual action is therefore

$$
\begin{aligned}
S^{\natural}=S_{0}[g] & +\frac{k}{4 \pi} \int_{\mathcal{M}} d z d \bar{z}\left[\frac{\lambda^{2}}{d_{11}} \partial \psi^{1} \bar{\partial} \psi^{1}-\frac{\lambda}{d_{11}} J_{1} \bar{\partial} \psi^{1}\right] \\
& +\frac{k}{4 \pi} \int_{\mathcal{M}} d z d \bar{z}\left[\frac{\lambda d_{\alpha 1}}{d_{11}} \partial \theta^{\alpha} \bar{\partial} \psi^{1}-\frac{\lambda d_{1 \alpha}}{d_{11}} \partial \psi^{1} \bar{\partial} \theta^{\alpha}\right] \\
& +\frac{k}{4 \pi} \int_{\mathcal{M}} d z d \bar{z}\left[\left(d_{\alpha \beta}-\frac{d_{\alpha 1} d_{1 \beta}}{d_{11}}\right) \partial \theta^{\alpha} \bar{\partial} \theta^{\beta}-\left(J_{\alpha}-\frac{d_{1 \alpha}}{d_{11}} J_{1}\right) \bar{\partial} \theta^{\alpha}\right] .
\end{aligned}
$$

It is believed that the dual theory is conformally invariant, provided that the dilaton is suitably transformed [5]. For the example we are considering here, we shall also see this more explicitly later.

If we integrate (2.10) by parts, only the boundary term contributes which is, on the torus, of the form

$$
\frac{\lambda k i}{4 \pi}\left(\Delta^{a} \psi^{1} \Delta^{b} \theta^{1}-\Delta^{b} \psi^{1} \Delta^{a} \theta^{1}\right)
$$

In the path integral we integrate over all $\theta^{1}$ satisfying (2.4) for $\alpha=1$. The group $G$ is simply-connected, and the different components in the integral, parametrised by the different 
(allowed) values for $\Delta^{c} \theta^{1}$, should therefore all give the same contribution. As only $\exp (i S)$ is relevant in the path integral, this implies that the dual action is only non-zero for $\psi^{1}$ satisfying (cf. Alvarez et.al. [3])

$$
\frac{\lambda k}{4 \pi} \Delta^{d} \psi^{1} \Delta^{m} \theta^{1} \in 2 \pi \mathbb{Z} .
$$

Before we start interpreting this dual theory, let us note that the duality transformation can be reversed by the inverse transformation

$$
\int D \psi^{1} \exp \left(\frac{\lambda k i}{4 \pi} \int_{\mathcal{M}} d z d \bar{z}\left(\bar{\partial} \psi^{1} \partial \tilde{\theta}^{1}-\partial \psi^{1} \bar{\partial} \tilde{\theta}^{1}\right)\right) \exp \left(i S^{\natural}\right) .
$$

Indeed, completing the square in the sum of the dual action and the generating functional for the second transformation, we find

$$
\begin{aligned}
S^{\natural}+ & \frac{\lambda k}{4 \pi} \int_{\mathcal{M}} d z d \bar{z}\left(\bar{\partial} \psi^{1} \partial \tilde{\theta}^{1}-\partial \psi^{1} \bar{\partial} \tilde{\theta}^{1}\right) \\
= & S_{0}[g]+\frac{k}{4 \pi} \int_{\mathcal{M}} d z d \bar{z} \frac{\lambda^{2}}{d_{11}}\left(\partial \psi^{1}+\frac{d_{\alpha 1}}{\lambda} \partial \theta^{\alpha}-\frac{J_{1}}{\lambda}+\frac{d_{11}}{\lambda} \partial \tilde{\theta}^{1}\right)\left(\bar{\partial} \psi^{1}-\frac{d_{1 \alpha}}{a} \bar{\partial} \theta^{\alpha}-\frac{d_{11}}{\lambda} \bar{\partial} \tilde{\theta}^{1}\right) \\
& +\frac{k}{4 \pi} \int_{\mathcal{M}} d z d \bar{z}\left[d_{11} \partial \tilde{\theta}^{1} \bar{\partial} \tilde{\theta}^{1}-J_{1} \bar{\partial} \tilde{\theta}^{1}\right]+\frac{k}{4 \pi} \int_{\mathcal{M}} d z d \bar{z}\left(d_{1 \alpha} \partial \tilde{\theta}^{1} \bar{\partial} \theta^{\alpha}+d_{\alpha 1} \partial \theta^{\alpha} \bar{\partial} \tilde{\theta}^{1}\right) \\
& +\frac{k}{4 \pi} \int_{\mathcal{M}} d z d \bar{z}\left[\left(d_{\alpha \beta}-\frac{d_{\alpha 1} d_{1 \beta}}{d_{11}}+\frac{d_{\alpha 1} d_{1 \beta}}{d_{11}}\right) \partial \theta^{\alpha} \bar{\partial} \theta^{\beta}-\left(J_{\alpha}-\frac{d_{1 \alpha}}{d_{11}} J_{1}+\frac{d_{1 \alpha}}{d_{11}} J_{1}\right) \bar{\partial} \theta^{\alpha}\right]
\end{aligned}
$$

which equals the original action. Furthermore, we also have

$$
\frac{\lambda k}{4 \pi} \Delta^{m} \psi^{1} \Delta^{d} \tilde{\theta}^{1} \in 2 \pi \mathbb{Z}
$$

and thus the periodicity of $\tilde{\theta}^{1}$ is the same as that of $\theta^{1}$, because of (2.15).

The term in the second line of (2.13) is topological and vanishes on world-sheets with trivial topology. Apart from this term we can identify the dual action (2.13) with the original action (2.7). Indeed, if we choose (without loss of generality) $\lambda=d_{11}$, and parametrise the group as

$$
g_{0}=e^{i \psi^{1} \hat{e}_{1}}\left(\prod_{\alpha=2}^{n} e^{i \theta^{\alpha} \hat{e}_{\alpha}}\right) g
$$

where

$$
\hat{e}_{\alpha}:=e_{\alpha}-\frac{\operatorname{Tr}\left(e_{\alpha} e_{1}\right)}{\operatorname{Tr}\left(e_{1} e_{1}\right)} e_{1} \quad \text { for } \alpha \geq 2, \quad \hat{e}_{1}:=e_{1},
$$

then the two actions coincide. However, the minimal monodromy of the field $\psi^{1}$ (for this choice of $\lambda$ )

$$
\Delta^{m} \psi^{1}=4 \pi \frac{d_{11}}{2 \lambda k}=\frac{2 \pi}{k}
$$


is in general different from the minimal monodromy of $\theta^{1}$ before (2.5). (The monodromy is only the same if $\theta^{1}$ is a long root, and $k=1$.) In general, the dual action therefore does not simply correspond to a redefinition of the original fields (or a Weyl transformation as advocated by Kiritsis [15]), but rather to a certain non-trivial orbifold [7].

The above topological term guarantees that the monodromy of the other fields is in the coroot lattice up to integral multiples of (2.20) 1 . To see this we write $\Delta^{c} \psi^{1}=n^{c} \Delta^{m} \psi^{1}$ with $c=a, b$ and $n^{a}, n^{b} \in \mathbb{Z}$; the topological term then becomes

$$
\frac{1}{2} \sum_{\alpha=2}^{n}\left(n^{a} \operatorname{Tr}\left(\Delta^{b} \theta^{\alpha} e_{\alpha} e_{1}\right)-n^{b} \operatorname{Tr}\left(\Delta^{a} \theta^{\alpha} e_{\alpha} e_{1}\right)\right) .
$$

As we calculate the partition function of the theory, i.e. the path-integral of $\exp \left(i S^{\natural}\right)$ over the torus, we have to sum over all $n^{a}, n^{b} \in \mathbb{Z}$. Then (2.21) will imply that only those states contribute, whose monodromy $\Delta^{c} \theta^{\alpha} \hat{e}_{\alpha}$ satisfies the above condition. This then means that the dual action describes the orbifold of the original theory which is induced by the subgroup generated by

$$
\exp \left(\frac{2 \pi i}{k} e^{1}\right)
$$

We should also mention that the precise from for the redefinition of the fields (2.19) is a matter of choice: we can integrate the original action (2.11) by parts to rewrite it as

$$
\begin{aligned}
S_{0}\left[g_{0}\right]=S_{0}[g] & +\frac{k}{4 \pi} \int_{\mathcal{M}} d z d \bar{z}\left[d_{11} \partial \theta^{1} \bar{\partial} \theta^{1}-J_{1} \bar{\partial} \theta^{1}\right] \\
& +\frac{k}{4 \pi} \int_{\mathcal{M}} d z d \bar{z}\left[+\sum_{\alpha \geq 2}\left(\left(1-m_{\alpha}\right) d_{1 \alpha} \partial \theta^{1} \bar{\partial} \theta^{\alpha}+\left(1+m_{\alpha}\right) d_{\alpha 1} \bar{\partial} \theta^{1} \partial \theta^{\alpha}\right)\right] \\
& +\frac{k}{4 \pi} \int_{\mathcal{M}} d z d \bar{z}\left[\sum_{\alpha, \beta \geq 2} d_{\alpha \beta} \partial \theta^{\alpha} \bar{\partial} \theta^{\beta}-\sum_{\alpha \geq 2} J_{\alpha} \bar{\partial} \theta^{\alpha}\right]
\end{aligned}
$$

where $m_{\alpha} \in \mathbb{Z}$ and we have ignored the topological terms. We can then do the same analysis as before and find that, apart from the new monodromy property $(2.20)$ for the field $\psi^{1}$ and some topological terms, the dual action agrees with the original action, where we have parametrised

$$
\hat{e}_{\alpha}:=e_{\alpha}-\frac{\left(1+m_{\alpha}\right) \operatorname{Tr}\left(e_{1} e_{\alpha}\right)}{\operatorname{Tr}\left(e_{1} e_{1}\right)} e_{1} \quad \text { for } \alpha \geq 2, \quad \hat{e}_{1}:=e_{1} \text {. }
$$

Again, this indicates that the characteristic feature of the duality transformation is the modification of the monodromy properties, rather than a redefinition of the fields.

\footnotetext{
${ }^{3}$ In this case there exists a free field construction for which $e_{1}$ is represented by a free boson [10, 13]. At least in the case of $\mathfrak{g}=\mathfrak{s u}(2)$, this free field theory is self-dual with respect to the ordinary duality transformation of a free boson on a circle 12 .

${ }^{4}$ This is automatically true if $k$ is a multiple of $d_{11}$, as $\Delta^{c} \theta^{\alpha} \hat{e}_{\alpha}$ is always in the coroot lattice up to $k / d_{11} \Delta^{m} \psi^{1} e_{1}$.
} 


\section{Quantisation of the dual action}

Let us now turn to a more detailed analysis of the dual action (2.13). To simplify notation, let us introduce the fields

$$
\phi^{\alpha}= \begin{cases}\psi^{1} & \text { if } \alpha=1 \\ \theta^{\alpha} & \text { if } \alpha \neq 1\end{cases}
$$

and denote

$$
\hat{d}_{\alpha \beta}=\operatorname{Tr}\left(\hat{e}_{\alpha} \hat{e}_{\beta}\right) \quad \hat{J}_{\alpha}=2 i \operatorname{Tr}\left(\partial g g^{-1} \hat{e}_{\alpha}\right) .
$$

The dual action is then, apart from the topological term which does not matter for the following discussion,

$$
\begin{aligned}
S^{\natural}\left[\phi^{\alpha}, g\right]=- & \frac{k}{4 \pi} \int_{\mathcal{M}} d z d \bar{z} \operatorname{Tr}\left(g^{-1} \partial g g^{-1} \bar{\partial} g\right)+\frac{k}{12 \pi} \int_{\mathcal{B}} d^{3} y \varepsilon^{i j k} \operatorname{Tr}\left(g^{-1} \partial_{i} g g^{-1} \partial_{j} g g^{-1} \partial_{k} g\right) \\
& +\frac{k}{4 \pi} \int_{\mathcal{M}} d z d \bar{z} \hat{d}_{\alpha \beta} \partial \phi^{\alpha} \bar{\partial} \phi^{\beta}-\frac{k}{2 \pi} \int_{\mathcal{M}} d z d \bar{z} i \bar{\partial} \phi^{\alpha} \operatorname{Tr}\left(\partial g g^{-1} \hat{e}_{\alpha}\right) .
\end{aligned}
$$

We now calculate the variation of the action with respect to a variation of $\phi^{\alpha}$ and $g$, and find

$$
\delta S^{\natural}=\frac{k}{2 \pi} \int_{\mathcal{M}} d z d \bar{z} \operatorname{Tr}\left(\left[i \delta \phi^{\alpha} \hat{e}_{\alpha}+e^{i \phi^{\beta} \hat{e}_{\beta}} \delta g g^{-1} e^{-i \phi^{\beta} \hat{e}_{\beta}}\right] \bar{\partial}\left(i \partial \phi^{\beta} \hat{e}_{\beta}+e^{i \phi^{\beta} \hat{e}_{\beta}}\left(\partial g g^{-1}\right) e^{-i \phi^{\beta} \hat{e}_{\beta}}\right)\right),
$$

which implies the equation of motion

$$
\bar{\partial} \mathcal{J}=0
$$

where

$$
\mathcal{J}=\left(i \partial \phi^{\alpha} \hat{e}_{\alpha}+e^{i \phi^{\beta} \hat{e}_{\beta}}\left(\partial g g^{-1}\right) e^{-i \phi^{\beta} \hat{e}_{\beta}}\right)
$$

If we also define

$$
\overline{\mathcal{J}}=\left(g^{-1} i \bar{\partial} \phi^{\alpha} \hat{e}_{\alpha} g+g^{-1} \bar{\partial} g\right)
$$

then

$$
\bar{\partial} \mathcal{J}=e^{i \phi^{\beta} \hat{e}_{\beta}} g \partial \overline{\mathcal{J}} g^{-1} e^{-i \phi^{\beta} \hat{e}_{\beta}}
$$

and thus $\partial \overline{\mathcal{J}}=0$ is equivalent to $(3.4)$. It is then clear that the two currents $\mathcal{J}(z)$ and $\overline{\mathcal{J}}(\bar{z})$ only depend on one of the two variables. In terms of $g_{0}$ they are simply given as

$$
\mathcal{J}=\partial g_{0} g_{0}^{-1} \quad \overline{\mathcal{J}}=g_{0}^{-1} \bar{\partial} g_{0},
$$

where

$$
g_{0}=e^{i \phi^{\alpha} \hat{e}_{\alpha}} g .
$$

Following Witten [20], we want to calculate the Poisson brackets of these currents. It is clear that the Poisson bracket of $\mathcal{J}$ with $\overline{\mathcal{J}}$ vanishes, and that the calculation for $\mathcal{J}$ and $\overline{\mathcal{J}}$ is essentially identical. Let us introduce

$$
\mathcal{J}^{a}(z)=\operatorname{Tr}\left(t^{a} \mathcal{J}(z)\right) \quad \mathcal{J}^{b}\left(z^{\prime}\right)=\operatorname{Tr}\left(t^{b} \mathcal{J}\left(z^{\prime}\right)\right)
$$


and likewise

$$
\overline{\mathcal{J}}^{a}(\bar{z})=\operatorname{Tr}\left(t^{a} \overline{\mathcal{J}}(\bar{z})\right) \quad \overline{\mathcal{J}}^{b}\left(\bar{z}^{\prime}\right)=\operatorname{Tr}\left(t^{b} \overline{\mathcal{J}}\left(\bar{z}^{\prime}\right)\right) .
$$

We then find

$$
\delta \mathcal{J}^{a}=\operatorname{Tr}\left(g^{-1} e^{-i \phi^{\alpha} \hat{e}_{\alpha}} t^{a} e^{i \phi^{\alpha} \hat{e}_{\alpha}} g \partial\left(g^{-1} i \delta \phi^{\alpha} \hat{e}_{\alpha} g+g^{-1} \delta g\right)\right),
$$

and derive, as in [20], the Poisson brackets

$$
\left\{\mathcal{J}^{a}, \mathcal{J}^{b}\right\}=-\frac{2 \pi}{k} \delta^{\prime}\left(z-z^{\prime}\right) \operatorname{Tr}\left(t^{a} t^{b}\right)+\frac{2 \pi}{k} \delta\left(z-z^{\prime}\right) \operatorname{Tr}\left(\left[t^{a}, t^{b}\right] \mathcal{J}(z)\right),
$$

and similarly for the bared currents. If we define now

$$
J^{a}(z)=-\frac{i k}{2 \pi} \operatorname{Tr}\left(t^{a}\left(i \partial \phi^{\alpha} \hat{e}_{\alpha}+e^{i \phi^{\beta} \hat{e}_{\beta}}\left(\partial g g^{-1}\right) e^{-i \phi^{\beta} \hat{e}_{\beta}}\right)\right)=-\frac{i k}{2 \pi} \mathcal{J}^{a}(z),
$$

and

$$
\bar{J}^{a}(\bar{z})=\frac{i k}{2 \pi} \operatorname{Tr}\left(t^{a}\left(g^{-1} i \bar{\partial} \phi^{\alpha} \hat{e}_{\alpha} g+g^{-1} \bar{\partial} g\right)\right)=\frac{i k}{2 \pi} \overline{\mathcal{J}}^{a}(\bar{z}),
$$

then these currents satisfy the Poisson bracket relations,

$$
\left\{J^{a}(z), J^{b}\left(z^{\prime}\right)\right\}=f^{a b c} J^{c}(z) \delta\left(z-z^{\prime}\right)+\frac{k}{4 \pi} \delta^{a b} \delta^{\prime}\left(z-z^{\prime}\right),
$$

and

$$
\left\{\bar{J}^{a}(\bar{z}), \bar{J}^{b}\left(\bar{z}^{\prime}\right)\right\}=f^{a b c} \bar{J}^{c}(\bar{z}) \delta\left(\bar{z}-\bar{z}^{\prime}\right)+\frac{k}{4 \pi} \delta^{a b} \delta^{\prime}\left(\bar{z}-\bar{z}^{\prime}\right)
$$

where $\left[t^{a}, t^{b}\right]=i f^{a b c} t^{c}$ and $\operatorname{Tr}\left(t^{a} t^{b}\right)=\frac{1}{2} \delta^{a b}$.

Next we want to expand the currents in modes, and in order to be able to do so, we have to analyse their monodromy. We can use the fact that the currents can be written in terms of $g_{0}$ as in (3.8), and that the monodromy of $g_{0}$ is $g_{0} \mapsto h_{p} g_{0}$, where

$$
h_{p}=\exp \left(2 \pi i \eta_{p} e^{1}\right) \quad \text { with } \quad \eta_{p}=\frac{p}{k},
$$

and $p$ is an integer. It is then easy to see that the monodromy of $\bar{J}^{a}(\bar{z})$ is trivial for each $p$, as

$$
\overline{\mathcal{J}}(\bar{z}) \mapsto g_{0}^{-1} h_{p}^{-1} \bar{\partial} h_{p} g_{0}=g_{0}^{-1} \bar{\partial} g_{0}=\overline{\mathcal{J}}(\bar{z}),
$$

and we can hence expand $\bar{J}^{a}(\bar{z})$ in modes

$$
\bar{J}^{a}(\bar{z})=\sum_{n \in \mathbb{Z}} \bar{J}_{n}^{a} \bar{\zeta}^{-n-1}
$$

where $\bar{\zeta}=\exp (2 \pi i \bar{z})$. Upon quantising the Poisson brackets, the modes then satisfy the (untwisted) Kac-Moody (KM) algebra

$$
\left[\bar{J}_{m}^{a}, \bar{J}_{n}^{b}\right]=i f^{a b c} \bar{J}_{m+n}^{c}+\frac{1}{2} k m \delta^{a b} \delta_{m,-n}
$$


For the following it is useful to write this algebra in a different basis, the modified Cartan-Weyl basis [14]. In this basis the algebra is generated by $\bar{H}_{m}^{i}, i=1, \ldots r, m \in \mathbb{Z}$, where $r$ is the rank of $\mathfrak{g}$, and $\bar{E}_{n}^{\alpha}, n \in \mathbb{Z}$, where $\alpha$ parametrises the roots. The commutation relations are given as (see for example [14]])

$$
\begin{aligned}
& {\left[\bar{H}_{m}^{i}, \bar{H}_{n}^{j}\right]=k m \delta^{i j} \delta_{m,-n}} \\
& {\left[\bar{H}_{m}^{i}, \bar{E}_{n}^{\alpha}\right]=\alpha^{i} \bar{E}_{m+n}^{\alpha}} \\
& {\left[\bar{E}_{m}^{\alpha}, \bar{E}_{n}^{\beta}\right]= \begin{cases}\varepsilon(\alpha, \beta) \bar{E}_{m+n}^{\alpha+\beta} & \text { if } \alpha+\beta \text { is a root, } \\
\frac{2}{\alpha^{2}}\left(\alpha \cdot \bar{H}_{m+n}+k m \delta_{m,-n}\right) & \text { if } \alpha=-\beta, \\
0 & \text { otherwise. }\end{cases} } \\
& {\left[k, \bar{E}_{n}^{\alpha}\right]=\left[k, \bar{H}_{n}^{i}\right]=0 .}
\end{aligned}
$$

Let us now turn to the mode expansion of the other current. Using the same reasoning as above, we see that the monodromy of $\mathcal{J}(z)$ is given by

$$
\mathcal{J}(z) \mapsto \partial\left(h_{p} g\right) g^{-1} h_{p}^{-1}=h_{p} \mathcal{J}(z) h_{p}^{-1} .
$$

If we write the various components of $J^{a}(z)$ in the modified Cartan-Weyl basis of the Lie algebra (corresponding to the above basis of the KM algebra), the adjoint action of $h_{p}$ is diagonal. For each sector described by the monodromy $h_{p}$, we can then expand the currents

$$
J^{i}(z)=\sum_{n \in \mathbb{Z}} H_{n}^{i} \bar{\zeta}^{-n-1}, \quad J^{\alpha}(z)=\sum_{m \in \mathbb{Z}+\left\langle\chi_{p}, \alpha\right\rangle} E_{m}^{\alpha} \bar{\zeta}^{-m-1}
$$

where $\zeta=\exp (2 \pi i z), \chi_{p}=\eta_{p} e_{1}$, and, to follow more conventional notation, $\langle\cdot, \cdot\rangle$ denotes the Killing form. Upon quantisation, these modes satisfy formally the same commutation relations as (3.21), but the indices $m$ are not all integral. This algebra is called the twisted KM algebra; the twist is inner and is given by (3.17).

We thus find that the dual model has various sectors which are parametrised by the monodromy of the field $\phi^{1}$. In each sector, the theory has two commuting infinite-dimensional symmetry algebras: the untwisted (right-moving) $\mathrm{KM}$ algebra $\bar{J}_{n}^{a}$ and the twisted (left-moving) KM algebra $H_{n}^{i}, E_{m}^{\alpha}$.

It is then clear, that the space of states is of the form

$$
\mathcal{H}^{\natural}=\oplus_{p} \mathcal{H}_{p}^{\natural},
$$

where $p$ denotes the different sectors, and each $\mathcal{H}_{p}^{\natural}$ is a subspace of

$$
\mathcal{H}_{p}^{\natural} \subset \mathcal{H}^{(p)} \otimes \mathcal{H}
$$

where $\mathcal{H}$ and $\mathcal{H}^{(p)}$ are the direct sums of all (irreducible) representations of the untwisted and the twisted KM algebra (with the twist corresponding to $h_{p}$ ), respectively. In general, we expect there to be different quantisations, which correspond to different (consistent) choices for the spaces $\mathcal{H}_{p}^{\natural}, c f$. [11]. However, as we shall show in the next section, there exists one quantisation for which the spectrum of the dual theory coincides with the original one, and this suggests that this theory is indeed equivalent to the original theory. 


\section{Representations of the twisted Kac-Moody algebra}

We now want to analyse the representation theory of the twisted KM algebra. The crucial property which will allow us to do this rather easily is the fact that the above twisted KM algebra is isomorphic to the untwisted algebra. This isomorphism is explicitly known, and can be defined as

$$
\begin{aligned}
\varphi\left(F_{m}^{\alpha}\right) & =E_{m+\left\langle\chi_{p}, \alpha\right\rangle}^{\alpha} \\
\varphi\left(I_{n}^{i}\right) & =H_{n}^{i}+k \chi_{p}^{i} \delta_{n, 0},
\end{aligned}
$$

where $H_{n}^{i}, E_{m}^{\alpha}$ are the generators of the twisted KM algebra corresponding to the $p$-th sector, and $I_{n}^{i}, F_{m}^{\alpha}$ are the generators of the untwisted KM algebra [14]. It is also clear, that the inverse exists, and that it is given by

$$
\begin{aligned}
\varphi^{-1}\left(E_{m}^{\alpha}\right) & =F_{m-\left\langle\chi_{p}, \alpha\right\rangle}^{\alpha} \\
\varphi^{-1}\left(H_{n}^{i}\right) & =I_{n}^{i}-k \chi_{p}^{i} \delta_{n, 0} .
\end{aligned}
$$

In particular, this implies that the representations of the twisted and the untwisted KM algebra are in one-to-one correspondence. We shall therefore denote the states in the corresponding representation spaces by the same symbol.

The untwisted KM algebra contains a Virasoro algebra

$$
\left[L_{m}, L_{n}\right]=(m-n) L_{m+n}+\frac{c}{12} m\left(m^{2}-1\right) \delta_{m+n, 0}
$$

in its universal enveloping algebra by virtue of the Sugawara construction, where the generators of the Virasoro algebra are given as

$$
L_{n}=\frac{1}{2 k+Q_{\psi}} \sum_{m \in \mathbb{Z}}\left(\sum_{i=1}^{r}: I_{n}^{i} I_{n-m}^{i}:+\sum_{\alpha} \frac{\alpha^{2}}{2}: F_{n}^{\alpha} F_{n-m}^{-\alpha}:\right) .
$$

Here $Q_{\psi}$ is the quadratic Casimir in the adjoint representation, : · denotes the usual normal ordering (see e.g. [14]), and

$$
c=\frac{k \operatorname{dim} \mathfrak{g}}{2 k+Q_{\psi}} .
$$

These generators are the modes of the stress-energy tensor, as

$$
\left[L_{m}, I_{n}^{i}\right]=-n I_{m+n}^{i} \quad\left[L_{m}, F_{n}^{\alpha}\right]=-n F_{m+n}^{\alpha} .
$$

Using the above isomorphism, it is then clear that the $\varphi\left(L_{n}\right)$ satisfy a Virasoro algebra with the same central charge, but that they are not quite the mode expansion of the stress-energy tensor as

$$
\begin{aligned}
{\left[\varphi\left(L_{m}\right), E_{n}^{\alpha}\right] } & =-\left(n-\left\langle\chi_{p}, \alpha\right\rangle\right) E_{m+n}^{\alpha} \\
{\left[\varphi\left(L_{m}\right), H_{n}^{i}\right] } & =-n H_{m+n}^{i}-k n \chi_{p}^{i} \delta_{m+n, 0}
\end{aligned}
$$


However, it is easy to see how to modify this by defining (c.f. for example [9])

$$
\begin{aligned}
L_{m}^{(p)} & =\varphi\left(L_{m}\right)-\chi_{p}^{i} H_{m}^{i}-\frac{1}{2} k \chi_{p}^{i} \chi_{p}^{i} \delta_{m, 0} \\
& =\varphi\left(L_{m}-\chi_{p}^{i} I_{m}^{i}+\frac{1}{2} k \chi_{p}^{i} \chi_{p}^{i} \delta_{m, 0}\right) .
\end{aligned}
$$

Then the $L_{m}^{(p)}$ define a Virasoro algebra with the same central charge as before, and

$$
\left[L_{m}^{(p)}, E_{n}^{\alpha}\right]=-n E_{m+n}^{\alpha} \quad\left[L_{m}^{(p)}, H_{n}^{i}\right]=-n H_{m+n}^{i} .
$$

In particular, this implies that the dual theory is a conformal field theory, as the theory contains a conformal stress-energy tensor.

We now want to analyse whether there exists a subspace $\mathcal{H}_{p}^{\natural}$ for each sector (labelled by $p$ ), so that the spectrum of the dual theory is the same as the original one. We observe that if we consider in the $p$-th sector only states which satisfy

$$
\frac{2}{\left\langle e_{1}, e_{1}\right\rangle}\left(e_{1} \cdot H_{0}\right) \mu=-p \mu,
$$

then

$$
\frac{2}{\left\langle e_{1}, e_{1}\right\rangle}\left(e_{1} \cdot I_{0}\right) \mu=p \mu
$$

and

$$
L_{0}^{(p)} \mu=L_{0} \mu
$$

as

$$
\begin{aligned}
\left(-\left(\chi_{p} \cdot I_{0}\right)+\frac{1}{2} k \chi_{p}^{2}\right) \mu & =\frac{p}{k}\left(-\left(e_{1} \cdot I_{0}\right)+\frac{\left\langle e_{1}, e_{1}\right\rangle}{2} p\right) \mu \\
& =\frac{p\left\langle e_{1}, e_{1}\right\rangle}{2 k}(-p+p) \mu=0 .
\end{aligned}
$$

This is not yet a complete solution, as we should restrict $p$ to lie in $0,1, \ldots, \frac{2 k}{\left\langle e_{1}, e_{1}\right\rangle}-1$, since the monodromy $h_{p}$ corresponding to $p$ and $p+\frac{2 k}{\left\langle e_{1}, e_{1}\right\rangle}$ is the same. (The coroot $2 e_{1} /\left\langle e_{1}, e_{1}\right\rangle$ is mapped to the unit element of the group under the exponential map.) On the other hand, in the spirit of orbifold constructions [7], we should expect that the states in each sector are characterised by their transformation property under the group of monodromies $\left\{h_{p}\right\}$. We should therefore consider in the $p$-th sector all states which satisfy

$$
\frac{2}{\left\langle e_{1}, e_{1}\right\rangle}\left(e_{1} \cdot H_{0}\right) \mu=-\left(p+\frac{2 k m}{\left\langle e_{1}, e_{1}\right\rangle}\right) \mu,
$$

where $m \in \mathbb{Z}$. Then we find that

$$
\frac{2}{\left\langle e_{1}, e_{1}\right\rangle}\left(e_{1} \cdot I_{0}\right) \mu=\left(p-\frac{2 k m}{\left\langle e_{1}, e_{1}\right\rangle}\right) \mu,
$$


and

$$
L_{0}^{(p)} \mu=L_{0} \mu+p m \mu .
$$

To see that the spectrum of $L_{0}$ is really the same, let us now recall that the affine Weyl group contains the subgroup of coroot translations, which act on the generators as [14]

$$
\begin{aligned}
I_{m}^{i} & \mapsto I_{m}^{i}(c):=I_{m}^{i}-k c^{i} \delta_{m, 0} \\
F_{n}^{\alpha} & \mapsto F_{n}^{\alpha}(c):=F_{n-\langle\alpha, c\rangle}^{\alpha} \\
L_{m} & \mapsto L_{m}(c):=L_{m}-c^{i} I_{m}^{i}+\frac{1}{2} k c^{i} c^{i} \delta_{m, 0},
\end{aligned}
$$

where $c$ is a coroot. If we apply this transformation with

$$
c=-\frac{2}{\left\langle e_{1}, e_{1}\right\rangle} m e_{1}
$$

to the above state, satisfying (4.14), we find that

$$
\frac{2}{\left\langle e_{1}, e_{1}\right\rangle}\left(e_{1} \cdot I_{0}(c)\right) \mu=\left(p+\frac{2 k m}{\left\langle e_{1}, e_{1}\right\rangle}\right) \mu,
$$

and

$$
\begin{aligned}
L_{0}(c) \mu & =\left(L_{0}+\frac{2}{\left\langle e_{1}, e_{1}\right\rangle} m\left(e_{1} \cdot I_{0}\right)+\frac{1}{2} k \frac{4}{\left\langle e_{1}, e_{1}\right\rangle} m^{2}\right) \mu \\
& =\left(L_{0}+m\left(p-\frac{2 k m}{\left\langle e_{1}, e_{1}\right\rangle}\right)+k m^{2} \frac{2}{\left\langle e_{1}, e_{1}\right\rangle}\right) \mu \\
& =L_{0} \mu+p m \mu .
\end{aligned}
$$

This is precisely the right-hand-side of (4.15). We can thus define $\mathcal{H}_{p}^{\natural}$ to be the span

$$
\mathcal{H}_{p}^{\natural}=\left\langle(\mu \otimes \nu) \in \mathcal{H}_{i} \otimes \mathcal{H}_{i} \mid \frac{2}{\left\langle e_{1}, e_{1}\right\rangle}\left(e_{1} \cdot I_{0}\right) \mu=\left(p+\frac{2 k m}{\left\langle e_{1}, e_{1}\right\rangle}\right) \mu ; m \in \mathbb{Z}, i \in R(\hat{\mathfrak{g}})\right\rangle,
$$

where $R(\hat{\mathfrak{g}})$ is the set of irreducible highest weight representations of the KM algebra $\hat{\mathfrak{g}}$, and $\mu$ is to be regarded as an element of the twisted representation space by virtue of the isomorphism (4.1). This gives rise to the consistent theory

$$
\mathcal{H}^{\text {dual }}=\bigoplus_{p=0}^{\frac{2 k}{\left\langle e_{1}, e_{1}\right\rangle}}-1
$$

as the definition respects the multiplicative structure of the field theory, i.e. the fusion product of states in $\mathcal{H}_{p_{1}}^{\natural}$ and $\mathcal{H}_{p_{2}}^{\natural}$ lies in $\mathcal{H}_{p}^{\natural}$, where $p=p_{1}+p_{2} \bmod 2 k /\left\langle e_{1}, e_{1}\right\rangle$. It is clear by construction, that this dual theory has the same spectrum as the original theory. 


\section{Conclusions}

We have analysed the (left-)chiral abelian duality transformation of a WZW model corresponding to a simple, simply-connected group. We have shown that the dual theory possesses two infinite dimensional symmetry algebras, a (right-moving) untwisted, and a (left-moving) twisted KM algebra. We have analysed the spectrum of this theory, and have shown that there exists a quantisation for which the spectrum of the original theory is recovered. This dual theory can naturally be interpreted as an orbifold [7] of the original model induced by the twists (3.17).

It would be interesting to do a similar analysis for various, more general cases: (i) the case, where the field $\theta^{1}$ with respect to which the transformation is performed is not purely left (or right) moving, and (ii) the case, where the fields $\theta^{i}$ span an arbitrary, not necessarily abelian subgroup of the target space group. (This latter case is usually referred to as non-abelian duality [2].)

It would also be interesting to understand which modifications arise if the target space group is not simply-connected.

\section{Acknowledgements}

It is a pleasure to thank Peter Goddard for many enlightening discussions. I also thank John Varghese for useful conversations at an early stage of this work, George Papadopoulos for helpful discussions, and Gérard Watts for a useful remark.

This work was supported by a Research Fellowship of Jesus College, Cambridge, and partly by PPARC and EPSRC, grant GR/J73322.

\section{References}

[1] Alvarez E., Alvarez-Gaumé L., Barbón J.L.F., Lozano Y.: Some global aspects of duality in string theory. Nucl. Phys. B 415, 71-100 (1994)

[2] Alvarez E., Alvarez-Gaumé L., Lozano Y.: On non-abelian duality. Nucl. Phys. B 424, 155-183 (1994)

[3] Alvarez E., Alvarez-Gaumé L., Lozano Y.: A canonical approach to duality transformations. Phys. Lett. B 326, 183-189 (1994)

[4] Alvarez E., Alvarez-Gaumé L., Lozano Y.: An introduction to T-duality in string theory. preprint CERN-TH-7486-94, hep-th/9410237

[5] Buscher T.: A symmetry of the string background field equations. Phys. Lett. B 194, 59-62 (1987); Path-integral derivation of quantum duality in nonlinear sigma-models. Phys. Lett. B 201, 466-472 (1988)

[6] Chu M., Goddard P., Halliday I., Olive D., Schwimmer A.: Quantisation of the WessZumino-Witten model on a circle. Phys. Lett. B 266, 71-81 (1991); Papadopoulos G., 
Spence B.: The canonical structure of Wess-Zumino-Witten models. Phys. Lett. B 292, 321-328 (1992)

[7] Dixon L., Harvey J., Vafa C., Witten E.: Strings on orbifolds. Nucl. Phys. B 261, 678-686 (1985); Strings on orbifolds (II). Nucl. Phys. B 274, 285-314 (1986)

[8] Felder G., Gawedzki K., Kupiainen A.: Spectra of Wess-Zumino-Witten models with arbitrary simple groups. Commun. Math. Phys. 117, 127-158 (1988)

[9] Freericks J.K., Halpern, M.B.: Conformal deformation by the currents of affine $g$. Ann. Phys. 188, 258-306 (1988)

[10] Frenkel I.B., Kac V.G.: Basic representations of affine Lie algebras and dual resonance models. Invent. Math. 62, 23-66 (1980); Segal G.: Unitary representations of some infinite dimensional groups. Commun. Math. Phys. 80, 301-342 (1981)

[11] Gaberdiel M.R.: WZW models of general simple groups. hep-th/9508105, DAMTP-9546, to appear in Nucl. Phys. B

[12] Ginsparg P.: Curiosities at $c=1$. Nucl. Phys. B 295, 153-170 (1988)

[13] Goddard P., Nahm W., Olive D., Schwimmer A.: Vertex operators for non-simply-laced algebras. Commun. Math. Phys. 107, 179-212 (1986)

[14] Goddard P., Olive D.: Kac-Moody and Virasoro algebras in relation to quantum physics. Int. Journ. Mod. Phys. A 1, 303-414 (1986)

[15] Kiritsis E.: Exact duality symmetries in CFT and string theory. Nucl. Phys. B 405, 109-142 (1993)

[16] Polyakov A.M., Wiegmann P.B.: Goldstone fields in two dimensions with multivalued actions. Phys. Lett. B 141, 223-228 (1984)

[17] Roček M., Verlinde E.: Duality, quotients, and currents. Nucl. Phys. B 373, 630-646 (1992)

[18] Seiberg N.: Electric-Magnetic duality in supersymmetric non-abelian gauge theories. Nucl. Phys. B 435, 129-146 (1995)

[19] Seiberg N., Witten E.: Electric-magnetic duality, monopole condensation, and confinement in N=2 supersymmetric Yang-Mills theory. Nucl. Phys. B 426, 19-52 (1994)

[20] Witten E.: Non-abelian bosonization in two dimensions. Commun. Math. Phys. 92, 455-472 (1984)

[21] Witten E.: String theory dynamics in various dimensions. Nucl. Phys. B 443, 85-126 (1995) 